\title{
Doubling the output power of dye-doped cholesteric liquid crystal lasers
}

\author{
Ying Zhou, Yuhua Huang, Alexandra Rapaport, Michael Bass, and Shin-Tson Wu \\ College of Optics and Photonics, University of Central Florida, Orlando, FL 32816
}

\begin{abstract}
Usually when optically pumped, dye-doped cholesteric liquid crystals (CLC) generate circularly polarized laser light from both directions of the lasing cell along the cholesteric helical axis. In reality, only the laser light from one direction can be utilized. In this paper, we demonstrate a simple method for doubling the laser output of a dye-doped CLC laser. The extracted laser output is nearly doubled. In experiment, we use a 6-ns, frequency-doubled Nd:YAG laser to pump the CLC lasing sample at $\sim 20$ degree incident angle. A reflector: a metal mirror or a cholesteric liquid crystal reflector is placed on the backside of the CLC sample. The reflector is in proximity contact with the CLC sample and the laser action occurs only in one direction. For the metal mirror reflector, the two orthogonal circularly polarized beams are mixed by incoherent superposition. While for the cholesteric liquid crystal reflector (same handedness as the lasing cell and highly reflective of the laser light), the enhanced laser output could also be achieved due to further stimulated amplification but the output is dominated by a single polarization state. For both cases the laser output is associated with a loss of coherence. Hence a nearly unpolarized CLC laser or a partially coherent CLC laser with nearly doubled output intensity is obtained.
\end{abstract}

Keywords: Cholesteric liquid crystals, Laser, Cholesteric liquid crystal reflector;

\section{INTRODUCTION}

Cholesteric liquid crystals have been widely used in liquid crystal full color displays, bistable displays, light shutters, tunable color filters and so on. In a typical CLC planar structure [1], the rod-like molecules are self-organized in a helical structure under certain surface alignment. When the linearly polarized light propagates through the medium, it experiences alternating ordinary and extraordinary refractive index. Hence the selective reflection band for circularly polarized light in the same handedness with cholesteric helix is built up if the number of periods is enough for Bragg reflection. Meanwhile, the opposite circularly polarized light will be highly transmitted. The reflection band can be easily determined by Eq.(1) and Eq.(2).

$$
\begin{aligned}
& \lambda_{0}=<\frac{n_{e}+n_{o}}{2}>\cdot p \\
& \Delta \lambda=\Delta n \cdot p
\end{aligned}
$$

where $\lambda_{0}$ and $\Delta \lambda$ represent the central wavelength of reflection band and the bandwidth of reflection band. $\Delta n, n_{e}, n_{o}, p$ represent birefringence, extraordinary refractive index, ordinary refractive index of liquid crystal, and the 
cholesteric intrinsic pitch length. Here $p$ is related with helical twist power (HTP) and the concentration $c \%$ of the chiral agent through Eq.(3):

$$
p=\frac{1}{H T P \cdot c \%}
$$

On the other hand, CLC is also regarded as chiral photonic medium [2]. Caused by anisotropic chiral structure, the photonic band gap exhibited in CLC broadens its way in photonic applications like lasers or optical diodes. Such a unique feature as periodic refractive index modulation resembles a 1 dimensional photonic crystal but with much easier fabrication process. Similar with a photonic crystal, localization of photons and shaping of density of states can be realized in cholesteric liquid crystals as well, which creates the possibilities of generating laser light for long-lived modes. Photonic band edge lasing in CLC was first theoretically analyzed by Dowling et al. in 1994 [3]. According to his work, the group velocity near the photonic band edge is real and tends toward zero, so the gain is greatly enhanced at the edge of the photonic band. In this case, CLC works as distributed feedback (DFB) cavity for stimulated emission. Afterwards lasing from CLC has been extensively investigated in both cholesteric liquid crystal host [4] and also in other host materials like ferroelectric liquid crystals [5], polymer network [6], and elastomers [7]. CLC based lasers have attracted considerable attentions not only because they are mirrorless but also because they have great tuning feasibility. As a result of sensitivity of helical pitch to external stimuli, tunable lasing can be controlled by external electrical field [8], temperature change [9], photo induced helical twisting power change [10] and $\mathrm{pH}$ change in chemical environments [11].

In CLC lasers, some of the previous work has been dedicated to improve the efficiency. It is generally believed that the order parameter of both dye's absorption and emission transition dipole moment plays important roles in determining the overall lasing efficiencies [12-13]. Hence it involves the correlation of many material parameters as birefringence, extraordinary refractive index and et al. But so far not much work discussed how to improve the extracted output efficiency from a device viewpoint. Due to the symmetry of CLC helical structure, when optically pumped, laser action is readily observed from both sides of cholesteric liquid crystal cell exactly along the cholesteric helix. In practical use, only the laser light from single direction can be fully utilized. In this paper, we incorporate a reflector to the lasing cell so that the laser light emitted can be extracted from single direction with doubled output. Two different types of reflector: a metallic mirror reflector or a cholesteric liquid crystal reflector is attached to the lasing cell and thus in both cases the output can be enhanced but with different polarization states. Correspondingly, either a nearly unpolarized CLC laser or a partially coherent CLC laser with enhanced output can be obtained by choosing different types of reflector. Physical mechanisms are also analyzed.

\section{SAMPLE PREPARATION}

The samples for our study were prepared by dissolving a highly emissive laser dye: 4-(dicyanomethylene)-2-methyl-6-(4-dimethlyaminostryl)-4H-pran (Exciton, DCM) at $2 \%$ weight concentration into CLC host. The CLC host is composed of nematic liquid crystal host BL006 (Merck, positive dielectric anisotropic) and $36 \%$ chiral agent CB15 (Merck, right handed). After thoroughly mixed, the whole mixture was capillary filled into a 15 $\mu \mathrm{m}$ thick cell which had ITO coating and polyimide alignment layers on both substrates. The alignment layers were 
rubbed in anti-parallel directions and the pretilt angle was small. A slowly cooling process gave rise to a cholesteric liquid crystal phase in planar structure. Due to the competition of the strong chiral turn and the surface anchoring energy, defect lines in CLC cell can not be completely eliminated. Hence scattering loss is introduced in the laser cavity, which limits the enhancement performance. Used as a CLC reflector, BL006 mixed with 34 \% CB15 without laser dye doped was filled into $5 \mu \mathrm{m}$ thin cell, shifting the reflection band to cover the lasing wavelength. According to our study, Bragg reflection can be well established in $5 \mu \mathrm{m}$ cell and moreover, a defect free cholesteric structure is maintained. Therefore there are not many advantages for further increasing the cell gap of the passive reflector.

\section{EXPERIMENTS AND RESULTS}

\subsection{Experimental setup}

We used the following experimental setup to study the emission properties of CLC laser incorporated with reflectors. As the pump source, Nd:YAG (Continuum, Minilite II) laser was operated at $\lambda=532 \mathrm{~nm}, 6 \mathrm{~ns}$ pulse width and $1 \mathrm{~Hz}$ repetition rate. The pump beam is split into two beams with one of them monitored by the energy meter (Ophir, Laserstar) and the other one focused into the lasing cell at 20 deg oblique incidence. Pump beam is changed into left handed circularly polarized light using a polarizer and a broadband quarter wave plate. Despite of dye molecules' absorption dichroism, dye-doped CLC doesn't show difference in the absorption of linearly polarized light or circularly polarized light. However, photonic band gap of CLC shifts to shorter wavelength with the increase of incident angle. Hence a circularly polarized pump beam in opposite handedness with cholesteric helix would help reduce the band gap reflection of the pump light as compared to using a linearly polarized pump. On the lasing cell, the pump beam spot was estimated around $150 \mu \mathrm{m}$ in diameter. A metallic mirror reflector, or a cholesteric liquid crystal reflector can be put in proximity contact to the backside of the lasing cell. The laser emission was collected by another lens into a fiber-based spectrometer (Ocean Optics, HR200 with resolution $=0.4 \mathrm{~nm}$ ). Here a CLC filter, exact the same as the CLC reflector we used, or a combination of a CLC filter and a broadband half wave plate can be used to detect the left-handed circularly polarized light (LCP) or right-handed circularly polarized light (RCP), respectively.

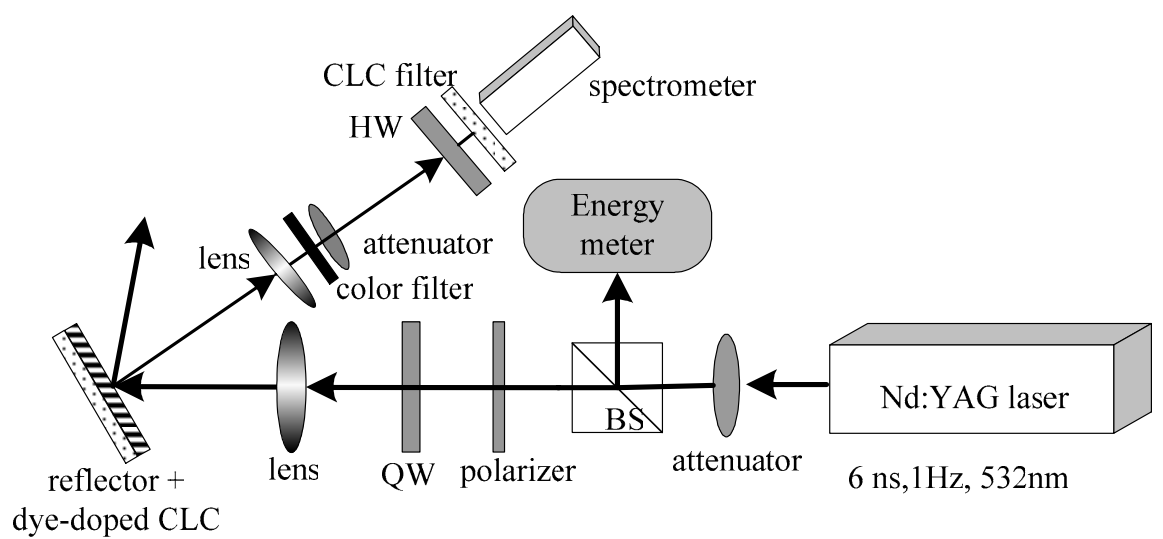

Fig.1. Experimental setup of a mirrorless CLC laser. BS: beam splitter, QW: quarter-wave plate, and HW: half-wave plate. 


\subsection{Experimental Results and Discussion}

Fig.2 describes the wavelength dependent normalized transmittance of dye-doped CLC lasing cell and the CLC reflector. Lasing spectrum from dye-doped CLC laser without reflector at pump energy $6.72 \mu \mathrm{J} /$ pulse is also included as an example. Centered at $\lambda=635 \mathrm{~nm}$, laser action occurs at long wavelength band edge of dye-doped CLC cell with full width of half maxima $(\mathrm{FWHM})=0.8 \mathrm{~nm}$. The short wavelength band edge is completely obscured by DCM absorption. Band gap of the CLC reflector is chosen to cover the lasing wavelength so that the output laser light facing toward the pump beam could be completely reflected back.

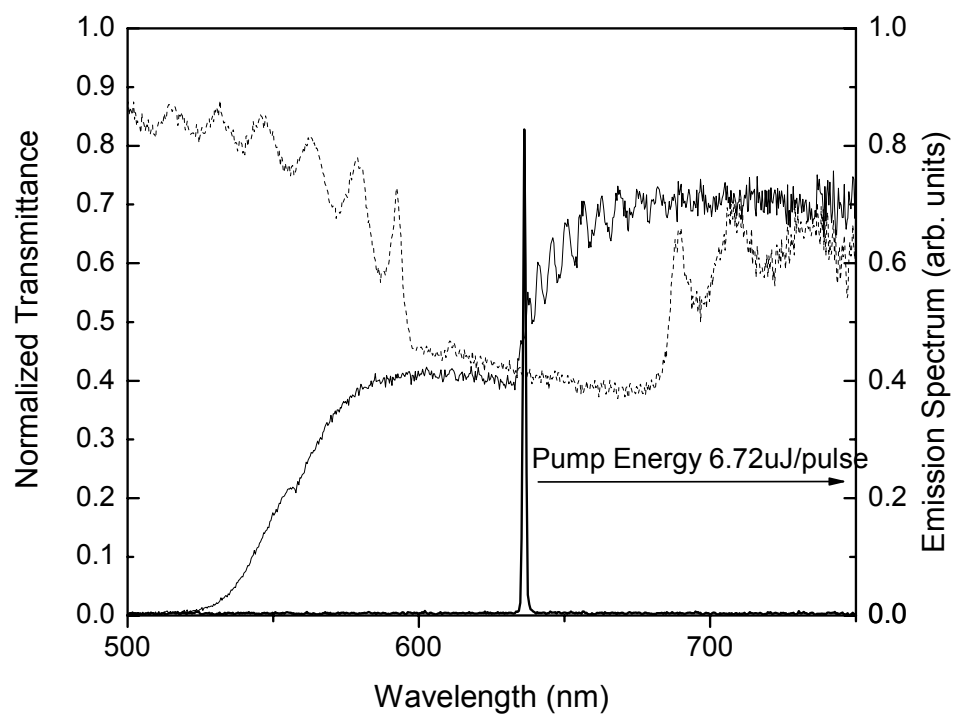

Fig.2 Transmittance and emission spectra of dye-doped CLC, and transmittance of CLC reflector. Thin solid and dash lines represent the transmittance of dye-doped CLC cell and CLC reflector, respectively. Thick solid line represents the lasing spectrum under left-handed circularly polarized pumping at $6.72 \mu \mathrm{J} /$ pulse and $20 \mathrm{deg}$ oblique incidence.

Emission properties of a single dye-doped CLC laser are investigated using Fig.1 setup and the results are shown in Fig.3. The solid line with filled squares represents the total output while the dash line with open triangles and solid line with filled circles are RCP component and LCP component, respectively. Lasing threshold is measured to be $\sim 1.3 \mu \mathrm{J} / \mathrm{pulse}$. It is seen that the laser emission is mainly RCP because for right-handed CLC structure, only RCP laser light experiences high reflectivity. Hence only RCP laser light gets feedback in the CLC based cavity. A small amount of LCP, occupying less than $10 \%$ of the total emission, is from the imperfection of cholesteric structure and the glass-air surface reflection. 


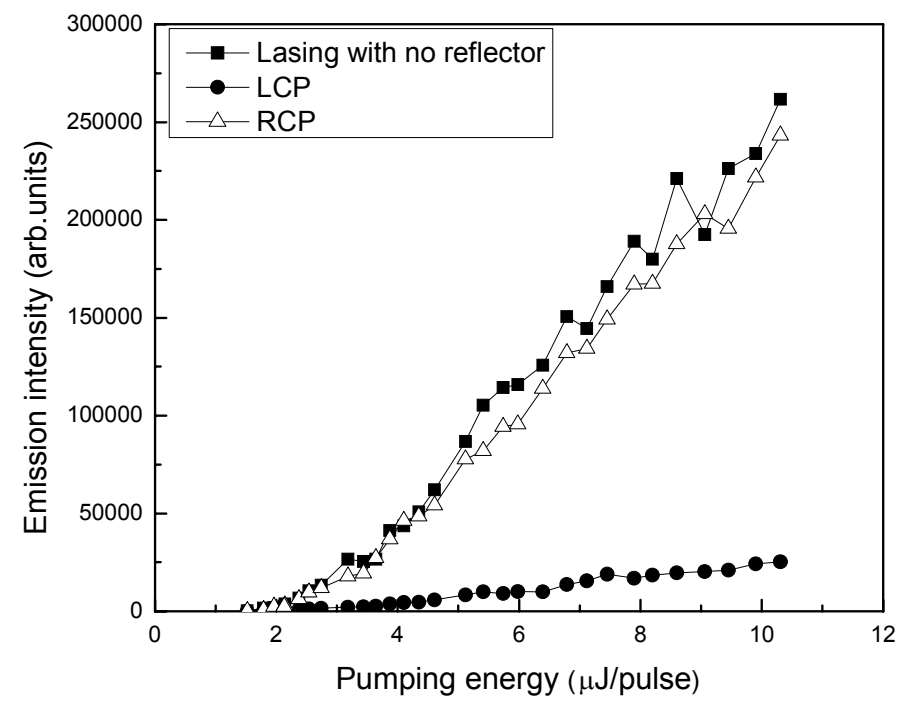

Fig.3 Output of a frequency doubled, Nd-YAG pumped, CLC laser with no reflector versus input energy. Closed squares are for the total output, open triangles are for the RCP output and closed circles are for the LCP output.

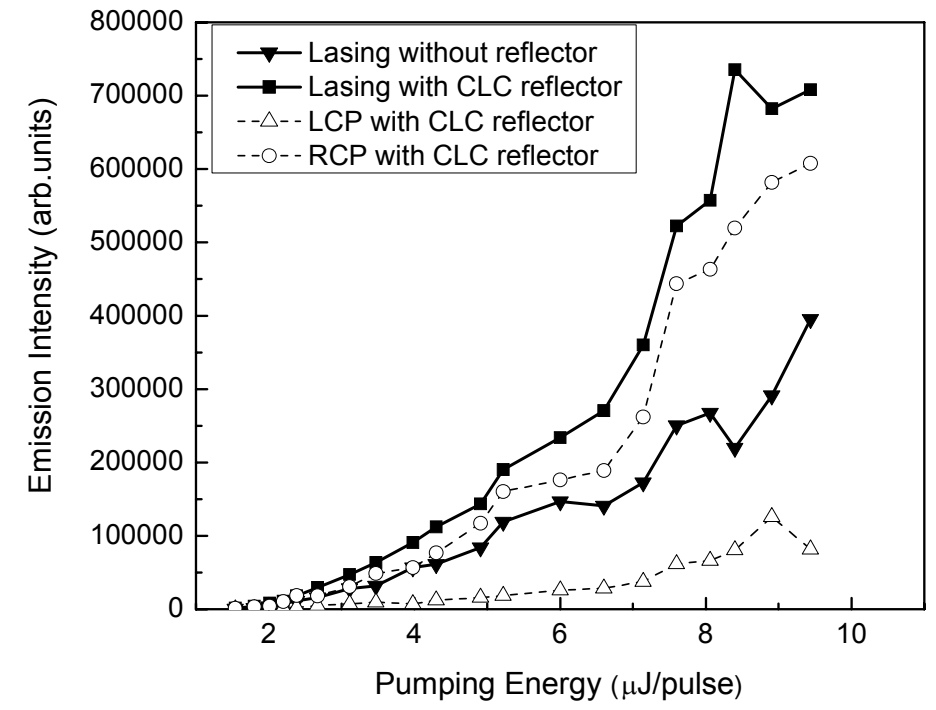

Fig. 4 Output intensity of the CLC laser with a CLC reflector versus input energy from the frequency doubled Nd:YAG laser. Solid lines with filled squares and filled triangles represent the total lasing intensity with and without a CLC reflector, respectively. Dashed lines with open triangles and open circles represent the LCP and RCP output intensity with a CLC reflector.

Fig.4 shows the pump energy dependent laser emission when a CLC reflector is placed in proximity contact with the downstream surface of CLC lasing cell. The total laser emission, RCP component and LCP component are shown in the solid line with filled squares, dash line with open circles and dash line with open triangles, respectively. The original 
laser emission from CLC laser without CLC reflector is also shown here in solid line with filled triangles. The arbitrary units on the ordinate scale are the same as used in Fig. 3 to allow an easy comparison.

Results show that the total laser intensity is increased by $\sim 2 \mathrm{X}$ and the RCP component dominates, which accounts for more than $85 \%$. At different pump energy, we observed a fluctuation in the enhanced laser output. This comes from: 1) the fluctuation of the pump beam, and 2) different refection points on the CLC reflector when different data points were taken. As shown in Fig.2, the CLC reflector is also right handed with whose reflection band chosen to reflect the lasing wavelength but transmit the pump wavelength. Based on Bragg reflection, upon reflection the polarization state of the reflected beam can be well preserved without appreciable change, namely RCP in our case. This reflected beam, at long wavelength edge of the reflection band and in the polarization state that is highly reflected by the lasing cell can be further amplified inside the cavity [14]. The incorporation of the CLC reflector to the master CLC lasing cell functions equivalently as a single CLC cell with increased DFB cavity length. A longer DFB provides larger optical gain amplification. As a result, the laser output is enhanced. However, the enhancement performance will be limited to $\sim 2 \mathrm{X}$ by the cavity loss, which mainly comes from the scattering of cholesteric defects. The two glass substrates form a glass layer in $2.2 \mathrm{~mm}$ thick. So the reflected beam gains optical path difference $O P D=2 n_{\text {glass }} d_{\text {glass }}=2 * 1.5 * 2.2 \mathrm{~mm}=6.6 \mathrm{~mm}$ when it is reflected back into the cavity. For the laser light with $\mathrm{FWHM}=0.8 \mathrm{~nm}$ and $\lambda=635 \mathrm{~nm}$, the coherence length is estimated $<1 \mathrm{~mm}$. Since its optical path difference is larger than the coherence length, the reflected part is somehow incoherent with the original part. As the result, the coherence of the enhanced laser light slightly deteriorates. Therefore a partially coherent CLC laser with output enhanced is obtained.

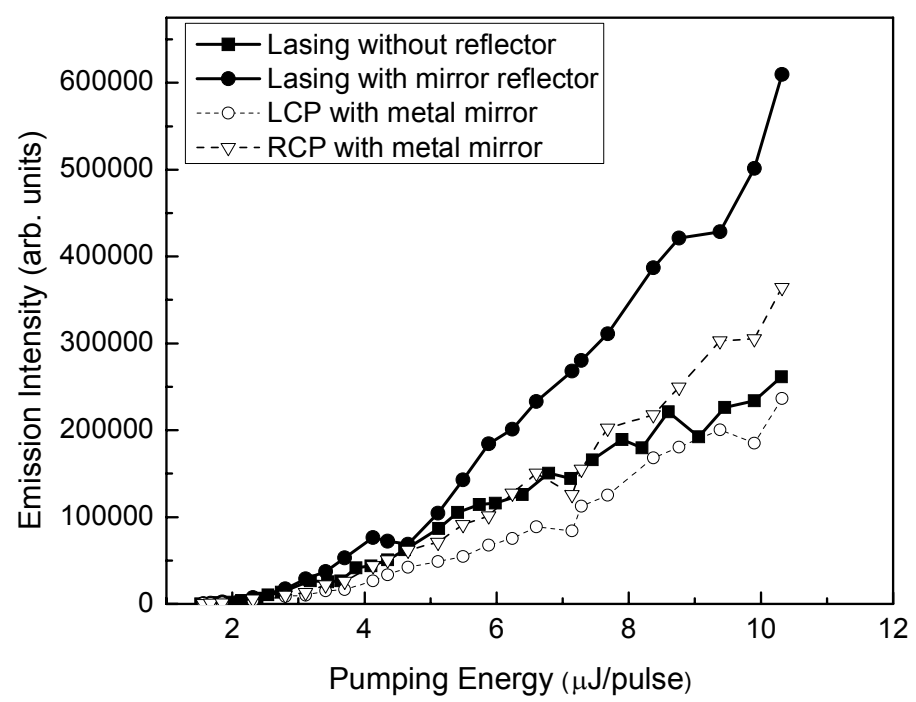

Fig.5 Pump energy dependent peak intensity of emission with a mirror reflector. Solid lines with filled circles and filled squares represent the total lasing intensity with and without the metal mirror reflector, respectively. Dashed lines with open circles and open triangles represent the LCP and RCP output intensities with the metal mirror reflector. 
Fig. 5 shows the improved performance on laser emission when a metallic mirror is attached. The solid line with filled circles, the dash line with open triangles and the dash line with open circles describe the total laser emission, RCP component and LCP component with mirror attached. The solid line with filled squares describes the original total laser output intensity. Results indicate that with mirror reflector attached the laser output is also increased by $\sim 2 \mathrm{X}$ but the polarization composition is different from the case of CLC reflector enhancement. Among the total output $\sim 60 \%$ is $\mathrm{RCP}$ component and $\sim 40 \%$ is LCP component. Because the metallic mirror brings in a $\pi$ phase change, when reflected back the original RCP reflected beam is changed into LCP component with some reflection loss involved. For the right handed CLC lasing cell, the reflected part will directly propagate through, contributing to the $40 \%$ LCP light in the total output. There is no obvious linear polarization direction observed in the total output laser. That means the original part and the reflected part are combined based on incoherent supposition. Hence by using a mirror reflector, a nearly unpolarized CLC laser is obtained.

\section{CONCLUSION}

In conclusion, we demonstrated a simple method to double the extracted laser output power in dye-doped cholesteric liquid crystal laser. At oblique incidence, a metallic mirror, or a cholesteric liquid crystal reflector with a reflection band covering the lasing wavelength is placed in proximity contact with the master lasing cell. By using a mirror reflector a nearly unpolarized CLC laser can be obtained based on incoherent supposition of two orthogonal circularly polarized beams. For cholesteric liquid crystal reflector, laser emission can be also doubled with single polarization state dominant but the coherence is sacrificed. Therefore we obtain a partially coherent CLC laser.

\section{REFERENCE}

1. S. T. Wu and D. K. Yang, Reflective Liquid Crystal Displays. (Wiley, New York, 2001)

2. V. I. Kopp, Z. Q. Zhang and A. Z. Genack, "Lasing in chiral photonic structures," Progress in Quantum Electronics 27, 369-416 (2003).

3. J. P. Dowling, M. Scalora, M. J. Bloemer, and C. M. Bowden, "The photonic band edge laser: A new approach to gain enhancement,” J. Appl. Phys. 75, 1896-1899(1994).

4. V. I. Kopp, B. Fan, H. K. M. Vithana, A. Z. Genack, "Low-threshold lasing at the edge of a photonic stop band in cholesteric liquid crystals," Opt. Lett. 23, 1707-1709 (1998).

5. M. Ozaki, M. Kasano, D. Ganzke, W. Haase, and K. Yoshina, "Mirrorless lasing in a dye-doped ferroelectric liquid crystal, , Adv. Mater. 14, 306-309 (2002).

6. T. Ohta, M. H. Song, Y. Tsunoda, T. Nagata, K. C. Shin, F. Araoka, Y. Takanishi, K. Ishkawa, J. Watanabe, S. Nishimura, T. Toyooka, and H. Takezoe, "Monodomain film formation and lasing in dye-doped polymer cholesteric liquid crystal,” Jpn. J. Appl. Phys. 43, 6142-6144 (2004).

7. H. Finkelmann, S. T. Kim, A. Munoz, P. Palffy-Muhoray, and B. Taheri, "Tunable mirrorless lasing in cholesteric liquid crystalline elastomers," Adv. Mater. 13, 1069-1072 (2001).

8. S. Furumi, S. Yokoyama, A. Otomo, and S. Mashiko, "Electrical control of the structure and lasing in chiral photonic band-gap liquid crystals," Appl. Phys. Lett. 82, 16-18 (2003). 
9. Y. Huang, Y. Zhou, and S. T. Wu, "Spatially tunable laser emission in dye-doped photonic liquid crystals," Appl. Phys. Lett. (In press, 2006).

10. A. Chanishvili, G. Chilaya, G. Petriashvili, R. Barberi, R. Bartolino, G. Cipparrone, and A. Mazzulla, L. Oriol, "Phototunable lasing in dye-doped cholesteric liquid crystals," Appl. Phys. Lett. 83, 5353-5355 (2003).

11. P. V. Shibaev, J. Madsen, A. Z. Genack, "Lasing and narrowing of spontaneous emission from responsive cholesteric films," Chem. Mat. 16, 1397-1399 (2004).

12. S. M. Morris, A. D. Ford, M. N. Pivnenko, and H. J. Coles, "Enhanced emission from liquid crystal lasers", J. Appl. Phys. 97, 023103 1-9 (2005).

13. K. C. Shin, F. Araoka, B. Park, Y. Takanishi, K. Ishikawa, Z. Zhu, T. M. Swager and H. Takezoe, "Advantages of highly ordered polymer dyes for lasing in chiral nematic liquid crystals," J. Appl. Phys. 43, 631-636 (2004).

14. Y. Zhou, Y. Huang, A. Rapaport, M. Bass, and S. T. Wu, "Doubling the optical efficiency of a chiral liquid crystal laser using a reflector,” Appl. Phys. Lett. 87, 231107 (2005). 